\title{
Effet du vieillissement sur quelques caractéristiques des manchons trayeurs
}

\author{
J. LE DU \\ avec la collaboration technique de Y. DANO \\ I.N.R.A., Laboratoire de Recherches sur la Traite \\ 65, rue de Saint-Brieuc, F 35042 Rennes
}

\section{Résumé}

L'effet du vieillissement sur les propriétés physiques et les caractéristiques de traite du manchon trayeur sont étudiés en comparant des manchons d'un même modèle à l'état neuf et après une utilisation de 1200 heures en ferme.

- Lorsque la pulsation est appliquée aux manchons pendant 100 heures, la tension des modèles neufs reste supérieure de 8 p. 100 à celle des modèles usagés. Par contre, les pressions de flambage, mesurées par le vide provoquant le contact entre les parois du manchon, sont identiques. Le vieillissement ne modifie pas l'aptitude du manchon à se plier sous le trayon.

- Pendant les 24 premières heures sous pulsation, la tension et la pression de flambage diminuent rapidement puis se stabilisent. Les crans prévus pour retendre les manchons apparaissent d'un intérêt très limité pour les modèles moulés.

- Les manchons sont comparés en trayant 24 vaches par demi-mamelle $(6$ vaches par semaine pendant 4 semaines). Avec les manchons usagés, on observe une faible diminution $(7,6$ p. 100) du volume d'égouttage $(\mathrm{P}<0,001)$ compensée par un accroissement du volume de lait machine $(\mathrm{P}<0,2)$. La souplesse de la lèvre plus élevée avec les manchons neufs $(2,56 \mathrm{~mm} / \mathrm{kg}$ contre $2,02 \mathrm{~mm} / \mathrm{kg})$ pourrait expliquer cet effet. Etant donné la similitude des pressions de flambage, les temps de traite et les débits ne sont pas affectés. Finalement, les performances de traite des manchons peuvent être maintenues pendant une durée importante $(1200$ heures, soit 3,5 heures de traite par jour pendant un an). Leur remplacement se justifie principalement par la difficulté de préserver une bonne propreté bactériologique avec des manchons trop usagés.

Mots clés: Machine à traire, manchon trayeur, vieillissement, caractéristiques physiques, élasticité.

\section{Introduction}

La durée d'utilisation des manchons trayeurs en caoutchouc synthétique est principalement limitée par la nécessité de préserver une hygiène de traite satisfaisante. En effet, l'absorption de matières grasses et la détérioration de l'état de surface rendent difficile le nettoyage et la désinfection (revue bibliographique de KeLLy, McKenNA \& O'SHEA, 1983). 
L'effet le plus tangible du vieillissement est de provoquer un «gonflement » du manchon et un relâchement de sa tension. Toutefois, les propriétés de l'élastomère telles que la dureté et le module d'élasticité (") sont peu affectées par une utilisation raisonnable - limitée à 1000 heures de traite, réparties sur un an - (RUFro \& SANGIORgI, 1976 ; Kelly et al., 1983 a).

En général, la détérioration ne devient sensible qu'après une utilisation anormalement prolongée. Ainsi, dans un cas d'utilisation intensive en ferme (8000 vaches traites en un an) Salvatierra. Hanson \& Kevey (1977) mettent en évidence une diminution hautement significative du module d'élasticité. Après 2370 heures réparties sur un an Ruffo \& Sangiorgi (1976) observent une diminution de 40 p. 100 de la résistance à la rupture sans que le module d'élasticité (*) soit modifié.

La comparaison des caractéristiques de traite des manchons neufs et usagés montre que l'incidence du vieillissement est faible par rapport à celle de la conception du manchon (forme et dimensions, Cicogna \& SANGIORGI, 1981). Avec des manchons usagés, correctement entretenus, la fréquence des chutes de gobelets est inchangée (Kelly et al., 1983 b) ou significativement diminuée (GibB \& MErN, 1976). Il est possible que cet effet soit dû à une meilleure adhérence du corps du manchon sur le trayon (Grbb \& MEIN, 1976). Par contre, si le vieillissement provoque une déformation visible de l'embouchure, les chutes de gobelets peuvent être fréquentes avec des manchons usagés (O'Shea \& O'CALlaGhan, $1980 \mathrm{c}$ ).

En ce qui concerne les paramètres caractérisant la traite, les différences significatives entre manchons sont en général en faveur des manchons usagés. Les productions de lait total et machine sont significativement accrues pour O'SHEA \& O'CALLAGHAN $(1980 \mathrm{c})$, après 2000 traites; pour KELLY et al. (1983 b) après 1000 heures. Ces derniers auteurs observent également une diminution du volume d'égouttage associée en général à un accroissement du temps de traite et exceptionnellement à une diminution de celui-ci.

Dans ce contexte, l'étude présentée a été entreprise pour tenter d'interpréter l'effet du vieillissement sur les caractéristiques de traite en fonction de la variation de certaines des propriétés physiques du manchon.

\section{Matériel et méthodes}

\section{A. Propriétés physiques des manchons}

Les mesures sont effectuées sur 6 manchons neufs et 6 manchons usagés de marque Alfa-Laval, modèle 96000001 , en néoprène. Les manchons usagés ont été utilisés pendant 1200 heures représentant environ 4000 traites de vaches en 13 mois. Le nettoyage a été effectué par la méthode dite acide et eau bouillante. Les manchons montés dans les gobelets au maximum de tension sont placés sur un banc d'essai et soumis à une pulsation continue pendant 100 heures (vide : $50 \mathrm{kPa}$, fréquence : $60 \mathrm{p} . / \mathrm{min}$; rapport : 50 p. 100.

(*) Pour la définition des propriétés mécaniques, voir P. Fournier (1968). 
Sur chaque manchon, la tension et la pression de flambage sont mesurées avec 4 répétitions, lors du montage puis après 15 minutes, 1 heure, 24 heures et 100 heures sous pulsation.

La tension $(\mathrm{kg})$ mesurée sur le manchon monté dans un gobelet en deux parties, est l'effort axial à appliquer à la moitié inférieure du gobelet pour la séparer de la moitié supérieure.

La pression de flambage $(\mathrm{kPa})$ est la mesure du vide à appliquer à l'intérieur du manchon monté dans son gobelet et fermé par un bouchon pour provoquer le contact entre les parois lors de l'applatissement.

Après 100 heures sous pulsation, l'élasticité de la lèvre est mesurée 2 fois sur chaque manchon. Une charge axiale est appliquée à un disque en plastique posé sur l'embouchure du manchon en faisant croître la charge de 0 à $400 \mathrm{~g}$. L'élasticité de la lèvre, exprimée en $\mathrm{mm} / \mathrm{kg}$ est appréciée par l'affaissement de la lèvre lorsque la charge atteint $400 \mathrm{~g}$ (LE Du, 1982).

\section{B. Caractéristiques de traite des manchons}

\section{Plan expérimental}

La comparaison des manchons s'effectue en trayant séparément chaque moitié du pis. Deux manchons neufs et deux manchons usagés sont montés de chaque côté d'un faisceau trayeur équipés de deux demi-griffes accolées (O'SHEA \& O'CALLAGHAN, $1980 \mathrm{~b}$ ).

Pendant chacune des 4 semaines de l'expérience, les caractéristiques de traite d'un lot de 6 vaches de race Française Frisonne Pie Noire sont contrôlées (24 animaux au total).

Chaque semaine, la position des manchons usagés et neufs est permutée après la traite du soir du $4^{\circ}$ jour de la semaine. Les contrôles ont lieu aux deux traites des jours 3, 4, 6 et 7 de la semaine.

\section{Matériel expérimental et critères mesurés}

La traite a lieu à $7 \mathrm{~h} 15$ et 16 h 15 dans une salle tandem équipée d'une ligne basse. La pulsation est simultanée : fréquence de $60 \mathrm{p} . / \mathrm{min}$; rapport de $72 \mathrm{p} .100$ et niveau de vide de $50 \mathrm{kPa}$. Le nettoyage est réalisé à l'aide d'un programmateur : $5 \mathrm{mn}$ de rinçage à l'eau froide, $15 \mathrm{mn}$ de nettoyage à $60^{\circ} \mathrm{C}$ avec une solution alcaline à $100 \mathrm{mg} / \mathrm{l}$ de chlore titrable, $5 \mathrm{mn}$ de rinçage à l'eau froide.

Les manchons neufs et usagés montés au maximum de tension sont mis sous pulsation pendant 24 heures avant d'être utilisés pour la traite. Les 2 manchons neufs sont changés chaque semaine et mis sous tension pendant la traite seulement. Les 2 manchons usagés ne sont jamais changés et demeurent sous tension dans les gobelets pendant les 4 semaines expérimentales.

Pendant la traite, le lait provenant de chaque demi-mamelle traverse un indicateur de fin de traite réglé pour allumer une lampe rouge lorsque le débit devient 
inférieur à $0,1 \mathrm{l} / \mathrm{mn}$. Le lait est ensuite recueilli dans un bocal de contrôle où est mesuré le volume de lait machine.

Pour chacun des deux circuits, le temps d'écoulement du lait machine est enregistré automatiquement. Après massage du pis, le trayeur pose les gobelets en enclenchant deux compteurs de temps électriques qui affichent le temps d'écoulement du lait machine quand le signal de fin de traite est émis.

Lorsque les deux lampes rouges sont allumées, le trayeur pratique un égouttage machine. Le lait est recueilli dans des bocaux différents de ceux utilisés pour le lait machine. Le volume de lait d'égouttage est ensuite mesuré dans une éprouvette graduée.

Il est alors possible de calculer :

- le débit moyen du lait machine $(l / m n)$ : volume de lait machine divisé par le temps d'écoulement du lait machine;

- le volume de lait total (l) : somme du volume du lait machine et du volume de lait d'égouttage.

\section{Résultats et discussion}

\section{A. Evolution des propriétés physiques des manchons}

Les résultats présentés à la figure 1 appellent essentiellement deux remarques :

- quel que soit le temps passé sous pulsation, la tension des manchons neufs demeure supérieure à celle des manchons usagés : 0,57 à $0,77 \mathrm{~kg}$, soit $8 \mathrm{p} .100$ en moyenne $(\mathbf{P}<0,001)$. L'allongement remanent provoqué par une utilisation de 1200 heures et peut-être la modification des propriétés de l'élastomère expliquent le relâchement irréversible de la tension des manchons usagés (Kelly, McKenNA \& O'SheA, 1983). Par contre, il n'existe aucune différence significative entre les pressions de flambage. Avec l'âge on sait que l'absorption de matières grasses accroît le volume du manchon. Il est donc possible que la diminution de la tension soit compensée par un accroissement du diamètre du corps signalé par Salvatierra \& Murphy (1975) ; Salvatierra, Hanson \& Kevey (1977); Kelly et al. (1983 a) et par un accroissement éventuel de l'épaisseur des parois. Ceci expliquerait la similitude des pressions de flambage des manchons neufs et usagés;

- quel que soit l'âge des manchons, pendant les 24 premières heures sous pulsation, la tension et la pression de flambage diminuent rapidement, respectivement de 8,7 p. 100 et 10,6 p. 100 cn moyenne. Ensuite, on atteint un certain état d'équilibre puisqu'entre 24 et 100 heures la décroissance moyenne est de 0,4 p. 100 pour la tension, de 4,2 p. 100 pour la pression de flambage.

Ce relâchement rapide de la tension était un inconvénient connu des anciens manchons extrudés en caoutchouc naturel qui devaient être fréquemment retendus dans leurs gobelets (Dodo \& Clough, 1959). Avec des manchons moulés, neufs ou usagés, nous observons le même phénomène. Pour le trayeur, la possibilité de retendre périodiquement le manchon à l'aide des crans prévus par le constructeur n'a qu'un intérêt très limité car les modifications - éventuelles — des conditions d'écoulement 


\section{TABLeau 1}

Comparaison des manchons neufs et usagés

(volumes de lait : exprimés par jour et par vache; temps et débits : moyennes par traite).

Comparison of new and used liners

(milk volumes : per day and per cow; time and flowrates : means per milking).

\begin{tabular}{|c|c|c|c|}
\hline & $\begin{array}{l}\text { Manchons neufs } \\
\text { New liners }\end{array}$ & $\begin{array}{l}\text { Manchons usagés } \\
\text { Aged liners }\end{array}$ & $\begin{array}{c}\text { Niveau } \\
\text { de signification }(*) \\
\text { Significance level }\end{array}$ \\
\hline $\begin{array}{l}\text { Lait total } \\
\text { Total milk }\end{array}$ & 19,43 & 19,45 & n.s. \\
\hline $\begin{array}{l}\text { Lait machine (1) ..... } \\
\text { Machine milk }\end{array}$ & 18,03 & 18,16 & $\mathbf{P}<0,2$ \\
\hline $\begin{array}{l}\text { Lait d'égouttage (l) } \ldots \ldots \\
\text { Stripping milk }\end{array}$ & 1,399 & 1,292 & $\mathbf{P}<0,001$ \\
\hline $\begin{array}{l}\text { Temps machine (s) ........ } \\
\text { Machine time }\end{array}$ & 199,4 & 202,4 & n.s. \\
\hline $\begin{array}{l}\text { Débit moyen }(1 / \mathrm{mn}) \ldots \ldots \\
\text { Mean flowrate }\end{array}$ & 2,74 & 2,71 & n.s. \\
\hline
\end{tabular}

(*) Test $\mathrm{t}$ par valeurs couplées (47 d.1.).

Paired observations $t$ test $(47$ d.f.).

du lait devraient s'estomper après 24 heures de traite au maximum (18 heures selon O'Shea \& O'Callaghan, 1980 a).

\section{B. Comparaison des caractéristiques de traite des manchons}

Avec les manchons usagés, nous observons une réduction significative de 7,6 p. $100 \mathrm{du}$ volume d'égouttage $(P<0,001)$ compensée par un accroissement du volume de lait machine $(\mathrm{P}<0,2)$ (tabl. 1). En comparant des manchons montés sur un même faisceau (traite par demi-mamelle), il existe a priori un risque de minimiser certaines différences liées, par exemple, aux stimulations d'origine mammaire ou aux chutes de gobelets. Toutefois, nos résultats sont globalement conformes aux conclusions obtenues en montant les manchons sur des faisceaux différents (GIBB \& MEIN, 1976 ; O’Shea \& O'Callaghan, 1980 c ; Kelly et al., 1983 b). Il est intéressant de noter que GibB \& MEIN (1976), en comparant 5 paires de manchons à l'aide d'un carré latin $10 \times 10$ observent que l'égouttage est en moyenne réduit de 7,7 p. 100 avec les modèles usagés sans que la réduction soit significative. Nous confirmons que l'intérêt du protocole expérimental utilisé (traite par demi-mamelle) réside dans la réduction de la variance des moyennes mesurées (O'SHeA \& O'CALlaGHAN, 1980 b). 


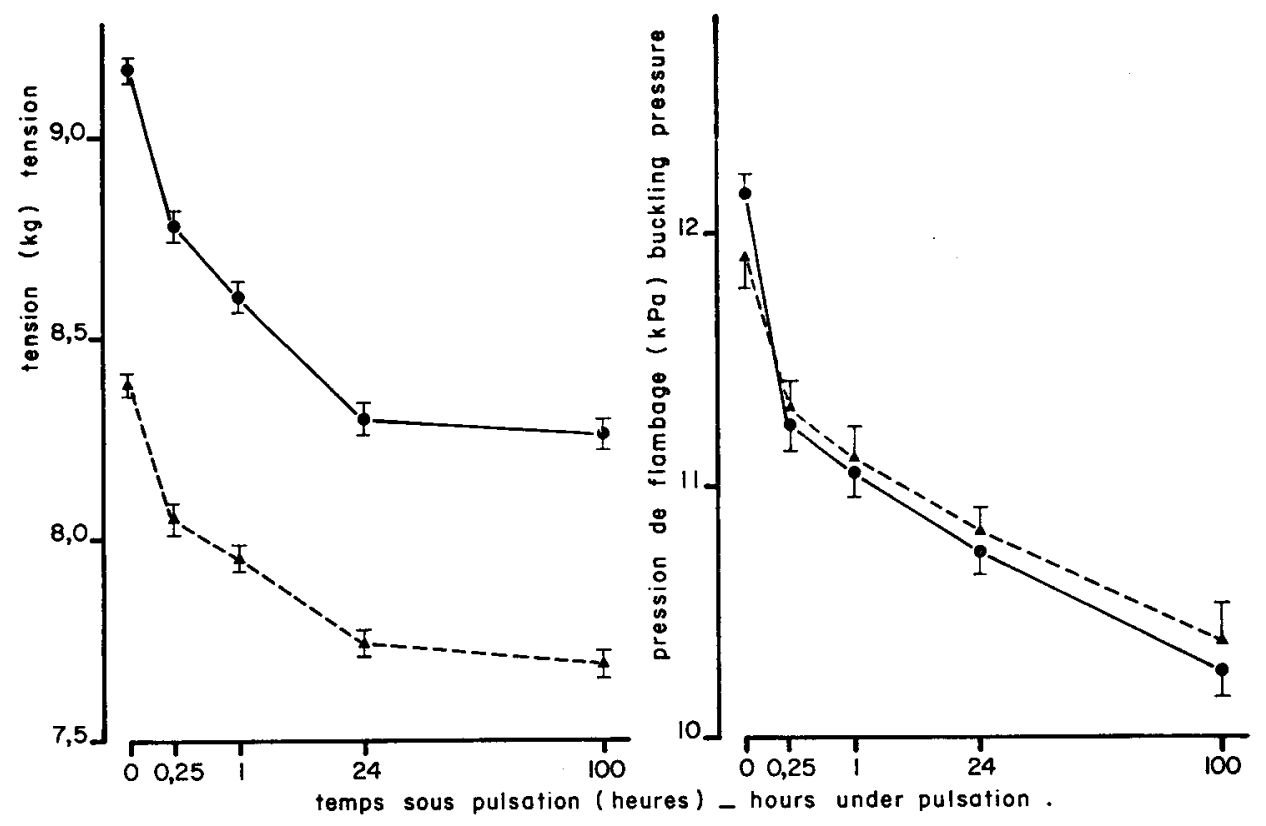

FIG. 1

Effet de l'application continue de la pulsation sur la tension et la pression de flambage de manchons neufs (traits pleins) et usagés (pointillé). Moyennes et erreurs types.

Effect of a continually applied pulsation on tension and buckling pressure of new liners (full line) and used liners (dotted line). Means and standard errors.

On admet que l'embouchure du manchon a une incidence sur l'égouttage tandis que le corps du manchon influence le début d'écoulement du lait (ThIEL \& MEIN, 1977). Dans nos conditions expérimentales, la souplesse de la lèvre est plus faible avec les manchons usagés $(2,02 \mathrm{~mm} / \mathrm{kg})$ qu'avec les manchons neufs $(2,56 \mathrm{~mm} / \mathrm{kg}$, $\mathrm{P}<0,001)$. Avec les manchons usagés, il est donc possible qu'un accroissement de friction entre la lèvre du manchon et la base du trayon permette une vidange plus complète du pis en réduisant le phénomène de "grimpage » du manchon contre le trayon (Mein et al., 1973). En fait, on dispose de peu d'éléments objectifs pour soutenir cette hypothèse car la souplesse de la lèvre est un paramètre qui n'est jamais mesuré. En conséquence, la diminution de l'égouttage avec les manchons usagés pourrait aussi bien être attribuée à un accroissement du diamètre de l'embouchure, observé par SAlvatierra, Hanson \& Kevey (1977) ou à une modification éventuelle de l'état de surface du corps du manchon, suggérée par GibB \& MEIN (1976).

Enfin, la similitude des temps de traite et des débits pour les manchons neufs et usagés s'interprète en considérant que les pressions de flambage sont identiques. Pour une pulsation de caractéristiques déterminées, on sait que ce paramètre détermine le temps pendant lequel le manchon est ouvert et que le lait s'écoule (LE Du, 1977). 


\section{Conclusions}

Il est habituel de justifier la réforme des manchons par les raisons suivantes : difficulté de nettoyage, diminution de «l'élasticité » et détérioration des caractéristiques de traite. En fait, si l'entretien est correctement effectué, une utilisation de 1200 heures n'altère pas l'aptitude du manchon à se plier sous le trayon (pression de flambage). Les caractéristiques de traite sont peu affectées par une utilisation relativement prolongée. Après 1200 heures de traite réparties sur 13 mois, le volume d'égouttage est plus faible qu'avec des manchons neufs sans que la production totale de lait ou le temps de traite soient affectés. Pour convaincre l'éleveur de changer périodiquement ses manchons trayeurs, il convient d'insister sur la difficulté de maintenir des manchons usagés en bon état de propreté bactériologique. Il est moins judicieux de faire état d'une éventuelle détérioration des conditions de traite d'autant plus que les éleveurs estiment en général que les manchons deviennent plus performants après un certain temps d'utilisation.

Du fait de leur conception, les anciens manchons extrudés devraient obligatoirement être montés sous tension. 11 était indispensable de les retendre périodiquement après avoir réduit leur longueur car l'absorption des matières grasses altère rapidement les propriétés du caoutchouc naturel. Avec les manchons monoblocs moulés en caoutchouc synthétique, les constructeurs continuent souvent à prévoir la possibilité d'ajuster la tension pour répondre à la demande de certains éleveurs. Cette conception du manchon est difficile à justifier car après 24 heures sous pulsation ( 3 heures de traite pendant 8 jours), la tension et la pression de flambage ont fortement diminué et demeurent ensuite relativement stables. On remarque d'ailleurs que la tension est très rarement ajustable sur les manchons moulés montés avec viseurs.

\section{Remerciements}

Nous exprimons notre reconnaissance au personnel de l'étable du Rheu pour la collaboration précieuse apportée à la réalisation de ce travail.

\section{Summary \\ Effect of ageing on some characteristics of teatcup liners}

The effect of ageing on some physical properties and milking characteristics of teat cup liners were studied comparing new and used liners (1 200 hours) of the same batch.

- When a pulsation was applied during 100 hours (fig. 1), the tension remained higher with new liners than with used ones $(8 \mathrm{p}$. 100). However, there were no differences for the buckling pressure measured by the vacuum which caused the contact between the two walls (touch point). Ageing did not affect the ability of a liner to collapse beneath the teat end.

- During the first 24 hours under pulsation there was a marked decrease in liner tension and buckling pressure which seemed to remain constant afterwards (fig. 1). Thus, with one piece liners made of synthetic rubber, periodic tension adjustment appeared of doubtful value.

- To study milking characteristics, liners were compared by the half udder technique on 6 cows per week during 4 weeks. With used liners, a slight $(7.6$ p. 100) but highly significant decrease in strip yield $(\mathrm{P}<0.01)$ was associated with an increase in machine milk $(\mathrm{P}<0.2)$ (tabl. 1). Possibly, this effect might be related to the lip collapsibility, higher 
with new liners $(2.56 \mathrm{~mm} / \mathrm{kg})$ than with used ones $(2.02 \mathrm{~mm} / \mathrm{kg})$. The lack of significant change in milking time and milk flow rates could be associated with the fact that buckling pressures are similar for both types of liners. This indicates that liners which are well looked after may maintain good milking characteristics after 1200 hours of utilization ( 3.5 hours per day, during one year). Thus the main reason for replacing liners is the difficulty of keeping a good bacteriological cleanliness in used liners.

Key words : Milking machine, liner, ageing, physical changes, elasticity.

Reçu en février 1984. Accepté en avril 1984.

\section{Références bibliographiques}

Cicogna M., Sangiorgi F., 1981. Caratteristiche di mungitura di alcune guaine del commercio nuove e usate. Zoot. Nutr. Anim., 7, 409-420.

Dodd F.H., Clough P.A., 1959. Use of the milking machine. In : Machine Milking. Minist. Agric. Fish. Fd Bull., 177, 66-68.

Fournier P., 1968. Les élastomères. In : Techniques de l'ingénieur, section A 2310, Paris, p. $1-20$.

GibB I.Mc D., MeIN G.A., 1976. A comparison of the milking characteristics of teatcup liners. Aust. J. Dairy Technol., 32, 148-153.

Kelly T.G., McKenna B., O'Shea J., 1983. Milking machine liners : a review of their chemical and physical characteristics and factors affecting their deterioration. In : Milking Machine Research at Moorepark, 1978-1982, An Foras Taluntais, Fermoy, Irlande, 14-23.

Kelly T.G., O'Callaghan E., McKenna B., O'Shea J., 1983 a. The use of a milking machine simulator to accelerate deterioration of milking machine liner. In : Milking Machine Research at Moorepark, 1978-1982, An Foras Taluntais, Fermoy, Irlande, 24-43.

Kelly T.G., O'Shea J., O'Callaghan E., Mckenna B., 1983 b. Comparison of milking characteristics of new and used liners. In : Milking Machine Research at Moorepark, 1978-1982, An Foras Taluntais Fermoy, Irlande, 44-55.

LE DU J., 1977. Machine à traire : paramètres physiques caractérisant le fonctionnement du manchon trayeur. Ann. Biol. Anim. Biochim. Biophys., 17, 971-985.

LE DU J., 1982. Comparaison de matériel de traite pour brebis : manchons en silicone et en caoutchouc, gobelets en inox ou en plastique. Ann. Zootech., 31, 139-148.

Mein G.A., Thiel C.C., Westgarth D.R., Fulford R.J., 1973. Friction between the teat and teatcup liner during milking. J. Dairy Res., 40, 191-206.

O'Shea J., O'Callaghan E., $1980 \mathrm{a}$. Milking performance of commercial clusters with standard pulsation. In : Experiments on milking machine components at Moorepark, 1976-1979, An Foras Taluntais, Fermoy, Irlande, 40-64.

O'Shea J., O'Callaghan E, $1980 \mathrm{~b}$. Milking performance of Half udder clusters. In : Experiments on milking machine components at Moorepark, 1976-1979, An Foras Taluntais, Fermoy, Irlande, 65-76.

O'Shea J., O'Callaghan E., $1980 \mathrm{c}$. Milking performance of full-udder clusters with standard pulsation : effect of cluster weight and cluster weight distribution and comparisons of original and imitation liners and new and used liners. In : Experiments on milking machine components at Moorepark, 1976-1979, An Foras Taluntais, Fermoy, Irlande, 104-114.

Ruffo G., SANGiorgi F., 1976. Machine milking : experimental observations on the life of teat-cup liners. Folia Veterinaria Latina, 6, 377-384.

Salvatierra S.A., MurPhy B.T., 1975. The effect of once and twice daily detergent cleaning on the deterioration of teat-cup liners. Aust. J. Dairy Technol., 30, 158-159.

Salvatierra S.A., Hanson W.J., Kevey W.A., 1977. A preliminary study on silicone teatcup liners. N.Z. J.P. Dairy Sci. Technol., 12, 238-241.

Thiel C.C., Mein G.A., 1977. Action of the cluster during milking. In : Machine Milking, N.I.R.D., Reading, Grande-Bretagne, 116-155. 